\title{
COMPACT 3-MANIFOLDS WITH INFINITELY-GENERATED GROUPS OF SELF-HOMOTOPY-EQUIVALENCES
}

\author{
DARRYL MCCULLOUGH ${ }^{1}$
}

\begin{abstract}
Examples are constructed of compact 3-manifolds with boundary whose groups of self-homotopy-equivalences are not finitely-generated.
\end{abstract}

For a finite CW-complex $X$, let $\mathscr{E}(X)$ denote the space of basepoint-preserving homotopy equivalences from $X$ to $X$, and let $\mathscr{G}(X)$ denote the group of homotopy equivalences $\pi_{0}(\mathscr{E}(X))$. An obvious question is: Under what conditions on $X$ must $\mathscr{G}(X)$ be finitely-generated? Sullivan [7] and Wilkerson [10] showed that if $X$ is simply-connected, then $\mathscr{G}(X)$ is finitely-presented. For non-simply-connected complexes, however, $\mathscr{G}(X)$ can be infinitely-generated (have no finite generating set) even for seemingly uncomplicated examples. Frank and Kahn [3] showed that $\mathscr{G}\left(S^{1} \vee S^{p} \vee S^{2 p-1}\right)$ is infinitely-generated when $p \geqslant 2$, and in [5] the author gave infinitely many examples of finite four-dimensional $K(\pi, 1)$-complexes with $\operatorname{Aut}(\pi)$ and, hence, $\mathscr{G}(K(\pi, 1))$ infinitely-generated. In [5], it was asked whether there was an example of an aspherical 2-complex $X$ with $\mathscr{G}(X)$ infinitely-generated. Recently, such examples were found by Brunner and Ratcliffe [2].

These various examples show two distinct ways that $\mathscr{G}(X)$ can fail to be finitely-generated. In the Frank and Kahn examples, $\pi_{2 p-1}\left(S^{1} \vee S^{p} \vee S^{2 p-1}\right)$ is quite large-it is infinitely-generated as a $\mathbf{Z} \pi_{1}\left(S^{1} \vee S^{p} \vee S^{2 p-1}\right)$-module-and many elements of $\mathscr{G}\left(S^{1} \vee S^{p} \vee S^{2 p-1}\right)$ arise by mapping the $S^{2 p-1}$ using an element of $\pi_{2 p-1}\left(S^{1} \vee S^{p} \vee S^{2 p-1}\right)$. In the aspherical examples, $\operatorname{Aut}\left(\pi_{1}(X)\right)$ is infinitely-generated and the asphericity forces $\mathscr{G}(X) \rightarrow \operatorname{Aut}\left(\pi_{1}(X)\right)$ to be surjective. Clearly, if $\mathscr{G}(X) \rightarrow \operatorname{Aut}\left(\pi_{1}(X)\right)$ is surjective then so is

$$
\mathscr{G}\left(X \vee S^{n}\right) \rightarrow \operatorname{Aut}\left(\pi_{1}\left(X \vee S^{n}\right)\right) \text { for } n \geqslant 2,
$$

so one can produce nonaspherical examples in dimension two. What is not apparent from these examples is the answer to the following question posed in [2].

Question. Is there a finite two-dimensional complex $X$ with $\operatorname{Aut}\left(\pi_{1}(X)\right)$ finitelygenerated but $\mathscr{G}(X)$ not finitely-generated?

Received by the editors July 29, 1983. This paper was presented June 21, 1982 at the Conference on Combinatorial Methods in Topology and Algebraic Geometry held at the University of Rochester.

1980 Mathematics Subject Classification. Primary 55P10, 57M99; Secondary 20H25.

Key words and phrases. Homotopy equivalence, self-homotopy-equivalence, 3-manifold, 2-complex. automorphism group, group ring.

${ }^{1}$ This research was supported in part by the National Science Foundation.

(1984 American Mathematical Society $0002-9939 / 84 \$ 1.00+\$ .25$ per page 
For a 3-manifold $M$ let $M^{\prime}$ denote the result of removing from $M$ the interiors of two disjoint closed 3-balls tamely-imbedded in the interior of $M$. Our main result is

THEOREM 1. Let $M$ be a compact aspherical 3-manifold-with-boundary, such that $\operatorname{Out}\left(\pi_{1}(M)\right)$ is finite and $\pi_{1}(M)$ admits a surjective homomorphism onto $\mathbf{Z} \times \mathbf{Z}$. Then $\mathscr{G}\left(M^{\prime}\right)$ is infinitely-generated.

Many instances of Theorem 1 are given in

COROllary. Let $M$ be a compact orientable 3-manifold-with-boundary such that the interior of $M$ admits a complete hyperbolic structure with finite volume, and such that $\pi_{1}(M)$ admits a surjective homomorphism onto $\mathbf{Z} \times \mathbf{Z}$. Then $\mathscr{G}\left(M^{\prime}\right)$ is infinitelygenerated.

Proof. $M$ is aspherical since its interior is, and $\operatorname{Out}\left(\pi_{1}(M)\right)$ is well known to be finite $[6$, p. $116 ; 8$, p. 5.31$]$.

For example, the (compact) complement of the Whitehead link and the (compact) complement of the Borromean rings are familiar 3-manifolds which satisfy the hypotheses of the theorem and the corollary.

Since any compact 3-manifold-with-boundary has the homotopy type of a finite 2-complex, and $\operatorname{Out}\left(\pi_{1}\left(M^{\prime}\right)\right)$ finitely-generated implies $\operatorname{Aut}\left(\pi_{1}\left(M^{\prime}\right)\right)$ finitely-generated, Theorem 1 answers the question of Brunner and Ratcliffe in the affirmative.

We will give the proof of Theorem 1 in $§ 1$, making use of two auxiliary theorems. These theorems, which are of independent interest, are proved in $\$ \S 2$ and 3. I wish to thank Andy Miller for helpful discussions concerning Theorem 2(b).

1. Proof of Theorem 1. Write $\pi$ for $\pi_{1}(M, *) \cong \pi_{1}\left(M^{\prime}, *\right)$. Let $\Phi: \mathscr{G}\left(M^{\prime}\right) \rightarrow \operatorname{Aut}(\pi)$ be the homomorphism defined by $\Phi(\langle f\rangle)=f_{\#}$. Let $\mathscr{G}_{1}\left(M^{\prime}\right)=\Phi^{-1}(\{1\})$ and $\mathscr{G}_{\text {Inn }}\left(M^{\prime}\right)=\Phi^{-1}(\operatorname{Inn}(\pi))$. Since $\operatorname{Out}(\pi)$ is finite, $\mathscr{G}_{\text {Inn }}\left(M^{\prime}\right)$ has finite index in $\mathscr{G}\left(M^{\prime}\right)$, so to prove the theorem it suffices to show $\mathscr{G}_{\operatorname{Inn}}\left(M^{\prime}\right)$ is infinitely-generated.

Let $M_{1}=M$ if $M$ is orientable, otherwise let $M_{1}$ be the orientable double cover of $M$. Now $M_{1}$ is compact, orientable, and has a boundary component which is not a 2-sphere. Therefore, $H_{1}\left(M_{1} ; \mathbf{Z}\right)$ is infinite so $M_{1}$ is sufficiently large. Therefore, the center of $\pi_{1}\left(M_{1}\right)$ is finitely-generated [9]. This implies that the center of $\pi$ is finitely-generated. Using Theorem 2(b), which will be stated and proved in $\S 2$, we see that $\mathscr{G}_{\text {Inn }}\left(M^{\prime}\right)$ is infinitely-generated if $\mathscr{G}_{1}\left(M^{\prime}\right)$ is.

Let Aut $_{\pi}\left(\pi_{2}\left(M^{\prime}\right)\right)$ be the group of $\pi$-module automorphisms of $\pi_{2}\left(M^{\prime}\right)$. We will prove that the natural homomorphism $\mathscr{G}_{1}\left(M^{\prime}\right) \rightarrow$ Aut $_{\pi}\left(\pi_{2}\left(M^{\prime}\right)\right)$ is surjective. Let $K$ be a finite 2-complex having the homotopy type of $M$; then $K$ is aspherical and $K^{\prime}=K \vee S^{2} \vee S^{2}$ has the homotopy type of $M^{\prime}$. Since $\pi$ has cohomological dimension two, the $k$-invariant $k\left(K^{\prime}\right)$ is zero. As shown in [2], this implies $\mathscr{G}_{1}\left(K^{\prime}\right) \rightarrow$ Aut $_{\pi}\left(\pi_{2}\left(K^{\prime}\right)\right.$ ) is surjective. (This surjectivity can be proved directly for $K^{\prime}$ without difficulty: just define a homotopy equivalence that is the identity on $K$ and induces the desired automorphism on $\pi_{2}\left(K^{\prime}\right)$.) Therefore, $\mathscr{G}_{1}\left(M^{\prime}\right) \rightarrow \operatorname{Aut}_{\pi}\left(\pi_{2}\left(M^{\prime}\right)\right.$ ) is surjective, so $\mathscr{G}_{1}\left(M^{\prime}\right)$ is infinitely-generated if $\operatorname{Aut}_{\pi}\left(\pi_{2}\left(M^{\prime}\right)\right)$ is. But $\pi_{2}\left(M^{\prime}\right) \cong \pi_{2}\left(K^{\prime}\right) \cong$ $\mathbf{Z} \pi \oplus \mathbf{Z} \pi$, so $\operatorname{Aut}_{\pi}\left(\pi_{2}\left(M^{\prime}\right)\right) \cong \mathrm{GL}_{2}(\mathbf{Z} \pi)$, the group of $2 \times 2$ invertible matrices 
with entries in $\mathbf{Z} \pi$. We apply Theorem 3, which will be stated and proved in §3, with $G=\pi$ to show that $\mathrm{GL}_{2}(\mathbf{Z} \pi)$ is infinitely-generated. This completes the proof of Theorem 1.

2. Proof of Theorem 2. While part (a) of Theorem 2 is not needed in the proof of Theorem 1, it is of independent interest and can be proved without much more work than that needed for part (b). A special case of part (a) appears in [4].

THEOREM 2. Let L be a finite-dimensional locally-finite connected simplicial complex. Then:

(a) If $\pi_{1}(L, *)$ is centerless, then $\mathscr{G}_{\mathrm{Inn}}(L) \cong \mathscr{G}_{1}(L) \times \pi_{1}(L, *)$.

(b) If the center of $\pi,(L, *)$ is finitely-generated, then $\mathscr{G}_{\mathrm{Inn}}(L)$ is infinitely-generated if and only if $\mathscr{G}_{1}(L)$ is infinitely-generated.

Proof. Write $\pi$ for $\pi_{1}(L, *)$. Replacing $L$ by the interior of a regular neighborhood of $L$, we may assume $L$ is a triangulated open manifold, and the basepoint $*$ is a vertex of $L$. Let $N$ be a regular neighborhood in $L$ of the 1-skeleton of $L$. Define $\alpha$ : $\pi \rightarrow \mathscr{G}_{\mathrm{Inn}}(L)$ as follows. For each $\sigma \in \pi$, choose an isotopy $H_{\sigma}: L \times I \rightarrow L$ starting at the identity map $1_{l}$ so that the trace of $H_{\sigma}$ (the homotopy class of the restriction of $H_{\sigma}$ to $\left.* \times I\right)$ equals $\sigma^{-1}$, and so that the restriction of $H_{\sigma}$ to $(L-\operatorname{int}(N)) \times\{t\}$ equals the identity for all $t \in I$. Let $h_{\sigma}(x)=H_{\sigma}(x, 1)$. Note that for $\tau \in \pi,\left(h_{\sigma}\right)_{\#}(\tau)$ $=\sigma \tau \sigma^{-1}$, so we can define $\alpha(\sigma)=\left\langle h_{\sigma}\right\rangle$.

We will now show that $\alpha$ is a homomorphism. For homotopies $G, H: L \times I \rightarrow L$ with $G(x, 1)=H(x, 0)$, we define $(G * H)(x, t)$ to be $G(x, 2 t)$ if $0 \leqslant t \leqslant \frac{1}{2}$ and to be $H(x, 2 t-1)$ if $\frac{1}{2} \leqslant t \leqslant 1$. We define $\bar{G}(x, t)$ to be $G(x, 1-t)$. Suppose $\sigma, \tau \in \pi$. Then $H_{\sigma} *\left(h_{\sigma} \circ H_{\tau}\right)$ is a homotopy from $1_{L}$ to $h_{\sigma} h_{\tau}$ with trace $\sigma^{-1} \cdot \sigma \tau^{-1} \sigma^{-1}=(\sigma \tau)^{-1}$. Therefore the trace of $\overline{H_{\sigma \tau}} *\left(H_{\sigma} *\left(h_{\sigma} \circ H_{\tau}\right)\right)$ is 1 so $\left\langle h_{\sigma \tau}\right\rangle=\left\langle h_{\sigma} h_{\tau}\right\rangle=\left\langle h_{\sigma}\right\rangle\left\langle h_{\tau}\right\rangle$.

Next, we will show that if $\langle f\rangle \in \mathscr{G}_{1}(L)$ and $\left\langle h_{\sigma}\right\rangle \in \operatorname{im}(\alpha)$, then $\langle f\rangle\left\langle h_{\sigma}\right\rangle=$ $\left\langle h_{\sigma}\right\rangle\langle f\rangle$. We may choose $f$ within its homotopy class so that $\left.f\right|_{N}=1_{N}$. A homotopy $G: f h_{\sigma} \simeq h_{\sigma} f$ is defined by $G(x, t)=h_{\sigma}(x)$ if $x \in N$ and $G(x, t)=H_{\sigma}(f(x), t)$ if $x \in L-\operatorname{int}(N)$.

Let $\mu: \pi \rightarrow \operatorname{Inn}(\pi)$ send $\sigma$ to the inner automorphism $\mu(\sigma)(\tau)=\sigma \tau \sigma^{-1}$. When $\pi$ is centerless, $\mu$ is an isomorphism and $\alpha \circ \mu^{-1}$ provides a splitting in the exact sequence $1 \rightarrow \mathscr{G}_{1}(L) \rightarrow \mathscr{G}_{\mathrm{Inn}}(L) \rightarrow \operatorname{Inn}(\pi) \rightarrow 1$. Since $\operatorname{im}(\alpha)$ commutes with $\mathscr{G}_{1}(L)$, this establishes part (a). For (b), observe that $\mathscr{G}_{1}(L) \cap \operatorname{im}(\alpha)$ is the image under $\alpha$ of the center of $\pi$, so it will be finitely-generated when the center of $\pi$ is. But inclusion induces an isomorphism $\mathscr{G}_{1}(L) /\left(\mathscr{G}_{1}(L) \cap \mathrm{im}(\alpha)\right) \cong \mathscr{G}_{\operatorname{Inn}}(L) / \operatorname{im}(\alpha)$, and (b) follows.

3. Proof of Theorem 3. Theorem 3 is proved by a modification of an argument of Bachmuth and Mochizuki.

THEOREM 3. Let $G$ be any group admitting a surjective homomorphism $\eta: G \rightarrow \mathbf{Z} \times \mathbf{Z}$. Then $\mathrm{GL}_{2}(\mathbf{Z G})$ is infinitely-generated. 
Proof. Let $s$ and $t$ be generators of $\mathbf{Z} \times \mathbf{Z}$, and denote the group ring $\mathbf{Z}[\mathbf{Z} \times \mathbf{Z}]=$ $\mathbf{Z}\left[s, s^{-1}, t, t^{-1}\right]$ by $R$. The homomorphism $\eta$ induces a homomorphism $\beta: \mathrm{GL}_{2}(\mathbf{Z} G)$ $\rightarrow \mathrm{GL}_{2}(R)$. Let $S=\mathrm{SL}_{2}(R) \cap \operatorname{im}(\beta)$.

We begin by using an idea from [2] to show that $\mathrm{GL}_{2}(\mathbf{Z G})$ is infinitely-generated if $S$ is. There is a short exact sequence

$$
1 \rightarrow \mathrm{SL}_{2}(R) \rightarrow \mathrm{GL}_{2}(R) \stackrel{\text { det }}{\rightarrow} R^{*} \rightarrow 1
$$

where the group of units $R^{*}$ is generated by $\{-1, s, t\}$. Let $R_{0} \subset R^{*}$ be the subgroup generated by $\left\{s^{2}, t^{2}\right\}$, which has index 8 in $R^{*}$, and let $H=\operatorname{det}^{-1}\left(R_{0}\right)$. If $w \in R_{0}$, then the "positive" square root $w^{1 / 2}$ is uniquely defined, and $f: H \rightarrow \operatorname{SL}_{2}(R)$ defined by $f(A)=(\operatorname{det}(A))^{-1 / 2} A$ is a retraction. Let $K=\operatorname{image}(\beta)$. Since $H$ has finite index in $\mathrm{GL}_{2}(R), K \cap H$ has finite index in $K$. But $\left.f\right|_{K \cap H}$ retracts $K \cap H$ onto $S$. Therefore, if $S$ is infinitely-generated, then so is $\mathrm{GL}_{2}(\mathbf{Z} G)$.

The proof that $S$ is infinitely-generated is a minor modification (for the case $P=\mathbf{Z}$ ) of the argument of $\S 2$ of [1] , and we use the notation of that paper. Choose $x, y \in G$ with $\eta(x)=s$ and $\eta(y)=t$. Lemma 1 of [1] is replaced by

LEMMA $1^{\prime} . E_{2}(R)$ is contained in

$$
\left(S \cap \mathrm{SL}_{2}\left(\mathbf{Z}\left[s, s^{-1}, t\right]\right)\right){ }_{V}\left(S \cap \mathrm{SL}_{2}\left(\mathbf{Z}\left[s, s^{-1}, t\right]\right)^{\left[\begin{array}{ll}
{\left[_{0}\right.} & 0 \\
1
\end{array}\right]}\right),
$$

where $V$ is the intersection of the factors.

LEMMA 2 is replaced by

LEMMA 2 . Let $\pi$ be a nonunit element of $\mathbf{Z}$. Then, the matrices

$$
\left[\begin{array}{cc}
1 & 0 \\
(s-1) / \pi^{i} & 1
\end{array}\right], \quad i \geqslant 1,
$$

can be chosen as part of a set of double coset representatives of $\left(S \cap \mathrm{SL}_{2}\left(Z\left[s, s^{-1}, t\right]\right)\right.$, $U)$ in $\mathrm{SL}_{2}\left(\mathbf{Q}\left[s, s^{-1}, T\right]\right)$.

The proof is unchanged. Alternatively, since

$$
S \cap \mathrm{SL}_{2}\left(\mathbf{Z}\left[s, s^{-1}, t\right]\right) \subseteq \mathrm{SL}_{2}\left(\mathbf{Z}\left[s, s^{-1}, t\right]\right),
$$

Lemma 2 implies Lemma 2'.

The argument continues exactly as in [1]. In the final calculation, one must check that

$$
M_{i}\left[\begin{array}{cc}
1 & \pi^{2 i} t^{-1} \\
0 & 1
\end{array}\right] M_{i}^{-1}=\left[\begin{array}{cc}
1-\pi^{i}(s-1) t^{-1} & \pi^{2 i} t^{-1} \\
-(s-1)^{2} t^{-1} & 1+\pi^{i}(s-1) t^{-1}
\end{array}\right]
$$

is in $S$. Let $D_{i}$ be this matrix, and let $D_{i}^{\prime}$ be the matrix

$$
D_{i}^{\prime}=\left[\begin{array}{cc}
1-\pi^{i} y^{-1}(x-1) & \pi^{2 i} y^{-1} \\
-(x-1) y^{-1}(x-1) & 1+\pi^{i}(x-1) y^{-1}
\end{array}\right]
$$


with entries in $\mathbf{Z} G$. Then $D_{i}^{\prime}$ is invertible with two-sided inverse

$$
\left[\begin{array}{cc}
1+\pi^{i} y^{-1}(x-1) & -\pi^{2 i} y^{-1} \\
(x-1) y^{-1}(x-1) & 1-\pi^{i}(x-1) y^{-1}
\end{array}\right]
$$

and $\beta\left(D_{i}^{\prime}\right)=D_{i}$.

\section{REFERENCES}

1. S. Bachmuth and $\mathrm{H}$. Mochizuki, $E_{2} \neq \mathrm{SL}_{2}$ for most Laurent polynomial rings, Amer. J. Math. 104 (1982), 1181-1189.

2. A. Brunner and J. Ratcliffe, Finite 2-complexes with infinitely-generated groups of self-homotopy equiralences, Proc. Amer. Math. Soc. 86 (1982), 525-530.

3. D. Frank and D. W. Kahn, Finite complexes with infinitely-generated groups of self-equivalences, Topology 16 (1977), 189-192.

4. J. Kalliongis, Homotopy equivalences on non-irreducible 3-manifolds, Indiana Univ. Math. J. 32 (1983), 903-915.

5. D. McCullough, Finite aspherical complexes with infinitely-generated groups of self-homotopy-equivalences, Proc. Amer. Math. Soc. 80 (1980), 337-340.

6. R. Riley, An elliptical path from parabolic representations to hyperbolic structures, Topology of Low-Dimensional Manifolds, Lecture Notes in Math., vol. 722, Springer-Verlag, Berlin and New York, 1979, pp. 99-133.

7. D. Sullivan, Infinitesimal computations in topolog1, Inst. Hautes Études Sci. Publ. Math. 47 (1977), 269-331.

8. W. Thurston, The geometry and topology of three-manifolds, mimeographed notes, Princeton University.

9. F. Waldhausen, Gruppen mit Zentrum und 3-dimensionale Mannigfaltigkeiten. Topology 6 (1967), 505-517.

10. C. W. Wilkerson, Applications of minimal simplicial groups, Topology 15 (1976), 111-130.

Department of Mathematics, The University of OKLahoma, Norman, OKlahoma 73019 